Voix et Images

volxetimages

\title{
Discours romanesque et discours urbain
}

\section{Guildo Rousseau et Lucie Grenier-Normand}

Volume 7, numéro 1, automne 1981

\section{Adrien Thério}

URI : https://id.erudit.org/iderudit/200306ar

DOI : https://doi.org/10.7202/200306ar

Aller au sommaire du numéro

\section{Éditeur(s)}

Les Presses de l'Université du Québec

ISSN

0318-9201 (imprimé)

1705-933X (numérique)

Découvrir la revue

Citer cet article

Rousseau, G. \& Grenier-Normand, L. (1981). Discours romanesque et discours urbain. Voix et Images, 7(1), 97-117. https://doi.org/10.7202/200306ar d'utilisation que vous pouvez consulter en ligne.

https://apropos.erudit.org/fr/usagers/politique-dutilisation/ 


\title{
Discours romanesque et discours urbain 1
}

\author{
par Guildo Rousseau et Lucie Grenier-Normand
}

La ville et le roman présentent deux discours culturels apparemment fort différents l'un de l'autre. D'abord géographique, le milieu urbain est fait d'espaces réels ou physiques (sol, climat, cours d'eau, aires boisées) et d'espaces fonctionnels ou d'usage (résidentiel, commercial, industriel, culturel), répartis sur un territoire donné. Le roman, lui, n'existe qu'en vertu d'un langage qui recrée ces espaces par des mots et des figures dont la portée dépasse nettement le sens usuel. Mais la ville est aussi un langage, un espace figurant et figuré, où s'enchevêtre tout un système de signaux, de signes et de symboles; non seulement se donne-t-elle à voir, mais elle nous communique une foule d'informations que nous recevons ou retenons selon nos réflexes, nos habitudes ou nos intérêts. Elle est, pour ainsi dire, un champ sémantique, un complexe d'images, mieux encore, un texte social: "L'image de la ville est semblable au mythe ou à l'œuvre littéraire», écrit Raymond Ledrut, «elle exprime et elle est exprimée ${ }^{2}$ ». La ville est à la fois histoire et récit.

II y aurait donc une certaine analogie entre le discours urbain et le discours littéraire. Du moins pourrait-on concevoir une certaine lecture de la ville, ou encore construire son image à partir des systèmes de communication qu'elle suggère aux citoyens ou qu'elle leur impose dans le but d'assurer son bon fonctionnement: code de la route, procès-verbaux des conseils de ville, rapports des commissaires de police, propos et confidences des citadins eux-mêmes sur leur rue ou leur quartier, leur façon d'animer leur ville, de l'investir ou de la subir. Autant de discours possibles sur la ville qu'il existe d'individus, de groupes ou de classes de citoyens, de réseaux d'institutions, qui vivent de la ville ou qui la font vivre.

Par ailleurs, le discours social et le discours littéraire échappent difficilement à la réciprocité qui les unit. Leur solidarité se noue dans un besoin mutuel de se dire ou de se raconter. Sans doute, ne peut-on jamais interchanger leur mode de représentation : le vécu urbain ne s'appréhende jamais de la même façon que le vécu romanesque; ni l'un ni l'autre ne s'invente non plus de la même manière comme imaginaire. Mais bien avant qu'elle ne soit publiée, l'œuvre littéraire est déjà un phénomène social : «elle contient déjà le public», comme l'a pressenti avec une grande finesse Gustave Lanson, voilà trois quarts 
de siècle ${ }^{3}$. Ainsi le discours social appelle le discours littéraire qui se presse de le nommer; mieux encore, l'un et l'autre se provoquent, se reprennent et échangent mutuellement leur mode de symbolisation. On serait même tenté de dire qu'ils s'imposent l'un à l'autre leurs différences: la ville fait ressortir la valeur onirique de ses lieux traditionnellement reconnus, tandis que le roman épuise la pratique signifiante de son discours. Dans cette perspective, l'expérience de l'espace urbain que décrivent les textes littéraires peut s'avérer révélatrice à plusieurs points de vue. Il se pourrait même que ce soit l'une des meilleures façons de comprendre le vécu urbain, tant « la ville apparaît beaucoup plus faite d'idées que de briques ${ }^{4}$, suivant l'excellente formule de Sylvie Rimbert.

Mais que peut-on apprendre du vécu urbain dans un corpus de romans dont le contenu réfère partiellement ou entièrement à une ville régionale? Voilà, en somme, la question qu'il nous apparaît essentiel de poser au début de cette analyse. Quelle symbolique de la ville peut-on en effet dégager d'une douzaine de romans ${ }^{5}$, parus entre 1925 et 1950, dont les paramètres de l'action se situent à l'intérieur des villes mauriciennes les plus importantes: TroisRivières, Shawinigan, La Tuque et Louiseville? Comment s'établit l'appropriation de la ville par les personnages? Quelle image rêvée de la ville est finalement à l'origine de l'antinomie presque naturelle entre la morphologie et la fonction de l'espace urbain, antinomie que les romanciers ne cessent d'exploiter, comme si elle était l'élément fondamental de leur discours sur la ville? Sans confondre le monde représenté et le monde représentant, ni non plus tracer entre les deux une frontière infranchissable, il convient de s'interroger sur l'échange ininterrompu de symboles, de signes et d'images récurrentes que les deux discours, urbain et littéraire, ont développé dans le roman mauricien entre les années 1925 et 1950 .

Analyser à fond l'échange réel qui s'est établi entre ces deux modes de discours à une époque donnée dépasserait d'emblée les limites du présent article. Tenter de reconstituer la vision du monde dans une œuvre littéraire, c'est, souvent, moins rechercher quelle partie de la réalité y est effectivement conservée ou retranchée, enrichie ou appauvrie, que saisir la portée de telles modifications du milieu social. Aussi limiterons-nous notre exposé à un seul niveau d'analyse: l'aménagement de l'espace urbain dans les romans retenus comme modèle particulier d'organisation du monde. Certains lieux du récit nous sont apparus comme des points d'articulation privilégiés dans la mise en scène de l'espace romanesque lui-même: l'église et le monastère, le collège et le couvent, l'usine de pâtes et papiers, la rue, le parc et la place du marché. C'est à partir de tels lieux, de leurs fonctions codantes, de leurs réseaux de relations avec les autres éléments du récit, de leurs possibilités symboliques et imageantes, que nous essaierons de reconstituer la signification de l'espace du texte. 


\section{L'échange symbolique}

\section{Le symbolisme des églises}

La représentation narrative de la ville mauricienne est avant tout dominée par le symbolisme des monuments religieux qui imposent leur inépuisable pouvoir de re-présentation. Lorsque les personnages vont vers l'une ou l'autre des villes de la Mauricie, ce sont d'abord les flèches élancées des hauts clochers des églises qui commandent leur regard et suscitent leurs premières réactions devant le paysage urbain.

Ainsi dans Trente arpents de Ringuet, les tours de la cathédrale de Trois-Rivières captent l'attention d'Euchariste Moisan bien avant qu'il n'atteigne les limites de la ville. Le paysan est pour ainsi dire arraché à la monotonie du voyage par la propriété exclusive de la vision qui se révèle à lui : l'aiguille du haut clocher, frappée par les rayons ardents du soleil, prend effectivement la forme "d'une colonne de lumière verticale ${ }^{6}$ ".

Mais Ringuet n'est pas le seul à exploiter un tel symbole de la verticalité. Bien avant lui, Adélard Dugré l'a utilisé de façon presque abusive dans La campagne canadienne, évoquant la modulation de voix célestes plutôt que l'harmonie des formes; avec lui, les clochers des églises de Trois-Rivières lancent au ciel leurs chants glorieux, auxquels se joignent ceux «de vastes établissements d'éducation et de charité», témoignant ainsi d'une foi catholique bien vivante ${ }^{7}$. La même impression de la verticalité, cette fois produite en sens inverse, séduit l'héroïne d'Eddie Hamelin, au moment où elle gravit "le long escalier à paliers" qui la conduit au monastère du Précieux-Sang : «À ses pieds s'étendait, ruisselante de blancheur et de soleil, la ville en activité. Les dômes du séminaire et du couvent des Ursulines se perdaient dans les clochers d'églises... ${ }^{8}$ \%.

Ces hauts clochers sont loin cependant de s'imposer par leur seule et unique signification religieuse. Suspendus entre ciel et terre, ils établissent entre les deux espaces une relation de causalité qui structure puissamment tout le paysage urbain. Du même coup l'opposition entre le ciel et la terre disparaît ; il n'y a plus qu'un réseau de figures symboliques qui ouvre l'espace et confère au paysage environnant son unicité, renvoyant à tous les clochers et à toutes les églises du territoire ${ }^{9}$, même au clocher natal, au lieu des ancêtres, et à l'églisemère de la collectivité ${ }^{10}$. Sans doute ne faut-il pas perdre de vue que les romans retenus ont tous été écrits avant la construction d'immeubles à multiples étages, tels la Place Royale ou la Place du Centre; entre 1925 et 1950 , la prédominance des monuments religieux «structurait» en quelque sorte le décor urbain. $\dot{A}$ eux seuls, ils assuraient une fidèle et paisible vigilance.

Écrits à une époque où la fréquentation des églises était habituelle, les romans ne décrivent pourtant le temple que de l'extérieur. Les personnages n'y pénètrent guère; ils n'osent franchir son parvis, comme si l'entrée dans ce lieu sacré leur était interdite, précisément parce qu'ils appartiennent à 
un univers profane. Ainsi percus comme des sentinelles, des remparts contre le monde, les temples urbains défient les hommes et les éléments de la nature. La cathédrale de Trois-Rivières, immuable et massive, force le piéton à la contourner et fait dévier la course du vent ". L'angle aigu de son clocher perce le ciel et déjoue la trajectoire des rayons solaires qui se réfléchissent sur ses hautes crêtes. La topographie des lieux s'établit encore par rapport à elle: les personnages habitent devant ou derrière la cathédrale, loin ou proche d'elle.

Mais la cathédrale de Trois-Rivières n'est pas un cas unique. A Louiseville, le héros du Poids du jour note, lui aussi, (lors d'un voyage) l'importance du nouveau temple dans le développement de sa ville natale; ses lignes anguleuses et massives inspirent même à Robert $M$. Garceau la nostalgie d'une époque où l'église de son enfance était plus familière, semblable à une vieille demeure au «dos voûté» et à la "peau rocailleuse»12.

Dans le panorama urbain, le temple domine par l'élévation de ses tourelles et de ses clochers. II est prestigieux, imposant, et par ses qualités architecturales, il s'affirme comme le symbole de la permanence du discours religieux. Mais à cette affirmation de force et de fierté se joint une certaine pointe d'agressivité et d'irritation. Un examen attentif du vocabulaire des romanciers permet de déceler l'influence quelque peu inhibitrice du monument religieux: "la flèche perçait", "piquait le bleu du ciel», "la ville était hérissée de clochers». Ces quelques traits laissent présager des conflits et des contraintes dans le paysage urbain.

\section{Les monastères et les chapelles}

Alors que les cathédrales et les églises s'impósent par leurs lignes architecturales et leur volume impressionnant, les monastères et les chapelles représentent plutôt des lieux propices à l'intériorisation et à l'expression de soi. Les personnages y retrouvent les attributs proprement féminins de la demeure: la paix intérieure, le réconfort moral, la pureté de l'enfance retrouvée, le courage renouvelé. La chapelle du couvent de La Tuque, celle de l'hôpital Saint-Joseph ou encore celle du Monastère du Précieux-Sang à Trois-Rivières, sont des centres nocturnes de quiétude. Le Monastère du Précieux-Sang surtout est source de renaissance spirituelle; par la perspective linéaire de ses longs corridors, par les longues allées de son sanctuaire, il symbolise la rencontre du ciel et de la terre. L'héroïne d'Eddie Hamelin est impressionnée «en pénétrant dans ce sanctuaire hospitalier " : elle «... sentit comme un grand attendrissement l'envahir tout entière. Elle voulut prier, mais la prière ne vint point. Le moment était pourtant propice, et le lieu, plus encore que son église paroissiale, invitait au recueillement et à l'élévation intérieure ${ }^{13}$. Comme toutes les chapelles décrites dans les romans étudiés, celle du Monastère du Précieux-Sang provoque des perceptions sensorielles intenses et envahissantes : les couleurs, les odeurs et les sons y sont subtils et délicats; le parfum discret de l'encens, le murmure des prières, les pas feutrés, les ombres silencieuses qui se glissent, les lueurs crépusculaires, tout respire 
la chaude et rassurante intimité ${ }^{14}$. Au paysage trifluvien, l'image du cloître et de la chapelle dépeinte dans les romans ajoute une dimension de profondeur par l'intensité de la vie intérieure qu'elle recèle. Ces deux lieux de prière jouent le rôle de nef, d'habitat parfaitement clos qui protège les hommes des vicissitudes de la vie terrestre.

\section{Les séminaires et les couvents}

D'autres édifices, tels les séminaires et les couvents sont reconnus pour leur prestige. Le Séminaire Saint-Joseph à Trois-Rivières est privilégié; cet édifice en impose à tous les personnages par «la tranquille harmonie de sa façade»; «le granit blanc» de ses «quatre colonnes toscanes" et les "armoiries du tympan», inspirent encore la fierté et «l'amour des traditions nationales » 15 , la confiance dans l'avenir aussi bien que la volonté de perpétuer les traditions morales et culturelles du milieu ${ }^{16}$. Par ailleurs, c'est également un lieu où s'affirme la différence des classes sociales. L'arrivée au séminaire du fils de paysan ne se fait jamais sans douleur; c'est une initiation qui suppose une mutation radicale, où le jeune homme doit s'arracher au monde qu'il a connu et aimé, à la terre dont il savait percer les secrets ${ }^{17}$.

Quant à l'image du couvent des filles, elle est aussi magique que prestigieuse. Un charme discrètement romantique en émane: "J'imaginais les couventines de la maison de briques s'échangeant leurs secrets", confie le héros de Pieds nus dans l'aube. Aux yeux du jeune homme, c'est encore un espace réservé, un peu tabou, plein de charme et de poésie: "Séparée de la rue par une rangée d'ormes et une mince clôture envahie de grimpants, la cour du couvent était un mélange de jardin, de promenade, d'oasis et de terrains de jeux ${ }^{18}$ ". Les diverses évocations des couvents pour jeunes filles permettent encore d'établir que ces maisons d'enseignement sont des centres d'éducation privilégiés. Ils évoquent le souvenir d'un passé où la discipline était de rigueur. lls éveillent même parfois un certain sentiment de culpabilité relié à une éducation rigide: "Vieux couvent toujours frais dont la présence imprévue

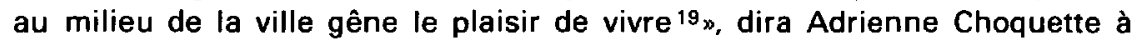
propos du couvent des Ursulines de Trois-Rivières.

A l'instar des collèges de garçons, les couvents de filles sont des monuments symboliques d'une influence considérable; les uns et les autres demeurent liés aux rythmes fondamentaux de la vie culturelle de l'époque 20 comme le sont dans un autre ordre les églises et les chapelles ${ }^{21}$, témoins de la foi religieuse. Sans doute la représentation symbolique de ces monuments n'est-elle rendue possible qu'à travers les impressions du romancier ou de ses personnages. Mais c'est justement parce qu'elle est traduite dans un langage, ou plus correctement par l'image littéraire d'un langage d'autrui (social), que nous pouvons y reconnaître une vision du monde. 


\section{Le symbolisme de la ville-usine}

Un espace profane encercle les monuments religieux organiquement liés au passé de la Mauricie, un espace discontinu, régi par la valeur économique, qui tend à remplacer par des signes neufs (presque exclusivement industriels) les symbolismes issus de la pratique sociale traditionnelle. Constamment, en effet, les romanciers opposent aux clochers des églises les longues cheminées des usines qui crachent dans le ciel mauricien leurs colonnes de fumée ${ }^{22}$. Cependant l'opposition est plus apparente que réelle : églises et usines imposent la même structure verticale qui génère la même variante symbolique. Au fur et à mesure que les personnages des romans se rapprochent du centre urbain, l'image de la ville-usine se fait de plus en plus précise, le regard est centré sur une aire déterminée: l'usine des pâtes et papiers, dont la vue suscite presque toujours les mêmes sentiments d'angoisse et de fascination.

Véritable siège des activités économiques de la région, l'usine des pâtes et papiers semble s'imposer tout autant que les monuments religieux et même davantage à la représentation littéraire de la ville mauricienne. Active dans presque tous les romans, elle établit pour ainsi dire son ascendant sur les personnages qui, de loin ou de près, se définissent presque toujours par rapport à elle. Avec ses hautes cheminées, ses chaînes sans fin et ses montagnes de «billots", elle surcharge aussi le paysage urbain d'une signification symbolique qui influe sur les relations de I'homme mauricien avec sa région.

En effet, elle est au centre du dur combat que se livrent la nature vierge et la ville industrielle, combat dont l'issue n'est malheureusement pas douteuse, du moins aux yeux des romanciers, puisqu'une même force obscure étend son pouvoir économique à la fois sur l'usine et sur la ville. Ce mouvement irréversible semble bien annoncer le passage, non sans nostalgie ni regrets, de la nature idyllique, de l'enfance insouciante, du travail artisanal, au monde de la conscience sociale, de la liberté assumée, du labeur mécanisé. "Je sortais de ma chère Vallée", confie le héros de Pieds nus dans l'aube, "comme les billots qui sortent par le Saint-Maurice et vont en ville se faire changer en papier ${ }^{23}$ ». Témoignage révélateur, qui exprime bien l'antithèse ville/forêt, mais aussi leurs réciproques relations; si la ville, comme l'écrit en effet Leclerc, “c'est le peuple rassemblé autour des usines ${ }^{24}$, celles-ci ont donné naissance à celle-là pour activer l'exploitation de la forêt. La petite ville de La Tuque n'échappe pas à la règle: "Tel le fruit le plus élevé d'un arbre immense", écrit encore Leclerc, «elle était la dernière de toute une série de petites villes semées comme des balises le long du Saint-Maurice 25 " et vivant de l'industrie du bois. La variété des termes employés par les romanciers pour désigner les usines de pâtes et papiers démontre bien la place que celles-ci occupent dans l'imaginaire mauricien: "fabrique de pâte de bois", "papeteries", "moulins à papier», «pulperies", «usines de pulpe», «industries du bois» et «manufacture de pulpe».

Mais la ville-usine apparaît surtout comme un monstre, une ogresse qui dévore les arbres. Empruntant leurs images tantôt à l'anatomie du corps 
humain, tantôt à la physiologie, les romanciers décrivent les villes mauriciennes comme un immense système digestif qui avale littéralement la forêt qui les entoure. Dans La Randonnée passionnée de Marie Lefranc, «un flot hoquetant» d'arbres arrachés à la forêt descend le Saint-Maurice «à un rythme de millions de billots par année» pour «nourrir» les «fabriques de pâtes de bois» de Grand-Mère ${ }^{26}$; dans $A$ la hache de Nantel, ces mêmes "spacieux moulins à papier " sont des «machines à dissection» qui «dépècent» sans arrêt la forêt mauricienne ${ }^{27}$. Hélas! cette "digestion" ne se fait pas sans misère et sans difficulté. L'énergie industrielle qui émane de la ville mauricienne et qui symbolise la prospérité matérielle acquise de haute lutte se révèle une force aliénante: les villes de La Tuque, de Grand-Mère et de Shawinigan sont soumises à la même domination technologique, à la même force économique qui était parvenue, à partir des années 1900, à dompter le cours tumultueux de la rivière Saint-Maurice:

Le fleuve avait fini par se laisser asseoir. Le grand vagabond des solitudes, avec son débit prodigieux, ses chutes, ses bassins faisait maintenant tourner les machines des centrales électriques des fabriques de pâte de bois, des papeteries... étonnantes de puissance et de modernisme... Et les villes champignons aux rues rectilignes, aux maisons fabriquées en série, aux hôtels, banques et bars que présidaient des hommes en manche de chemise, le brûle-gueule à la bouche, le chapeau rabattu sur les yeux, avaient surgi sur ses bords ${ }^{28}$.

Dans Trente arpents, c'est avec un réalisme cru, presque brutal, que le romancier traduit la surprise d'Euchariste Moisan devant le secteur industriel de Trois-Rivières: "La route maintenant se butait durement à une montagne de bois, toute une forêt dépecée en billes et écroulée sur la route. Cela faisait un barrage qui la déviait brutalement, comme ceux du Nord où les tronches enchevêtrées bloquent les rivières et les bousculent hors de leur lit ${ }^{29}$. » Plus encore, l'atmosphère suffocante provoque chez le paysan la sensation d'un malaise physiologique insupportable: «Et sur toute la ville s'appesantissait comme une malédiction une buée lourde et rance, piquante au nez, vomitive. Cette odeur de l'acide décomposant le bois, il la connaissait pour l'avoir sentie parfois jusque chez lui, quand le vent du nord-est visait juste Saint-Jacques. Mais jamais si violente, si écceurante ${ }^{30}$."

La multiplication des usines de pâtes et papiers, disséminées dans le paysage urbain mauricien, ferait donc obstacle à toute relation familière avec la ville. Ces usines-organes, aux viscères mal connues, deviennent même une source d'angoisse collective; du moins peut-on déceler chez les personnages un certain inconfort moral devant ces gigantesques ventres de béton destinés à la digestion de la forêt mauricienne, dont les activités impliquent dramatiquement une conception différente de la vie et de l'organisation de l'espace humain dans un milieu. Plus encore que les monuments religieux qui imposent par leur dehors un sens du sacré, les usines de pâtes et papiers, toutes profanes qu'elles soient, se chargent d'un mystérieux pouvoir symbolique: elles commandent et interdisent, elles répartissent les individus en 
classes sociales, rapprochant les uns, écartant les autres ${ }^{31}$, et rendent ainsi extrêmement difficile l'appropriation du paysage urbain. En quête de leur ville, les personnages du roman mauricien se sentent menacés par l'ordre que l'on tente de leur imposer. Ils éprouvent la sensation de parcourir leur ville sans la considérer réellement comme un milieu de vie.

\section{Les langages communicatifs de la ville}

Les personnages mauriciens partent habituellement à la conquête de leur ville en déambulant dans les rues, selon leur fantaisie, ce qui leur permet d'entrer en communication avec autrui, d'établir des échanges, d'accéder au rituel de l'usage spatial. La rue, lieu de passage permanent, lieu d'activités diversifiées, sert aussi d'observatoire au spectateur, mais elle ne se laisse pas facilement saisir dans sa totalité; espace à la fois privé et public, elle doit être considérée comme un univers constitué d'éléments hétérogènes où l'arbitraire des signes n'empêche pas l'expressivité des images. Aussi, vivre la ville, sa ville, c'est en quelque sorte s'approprier son langage ou plutôt ses multiples langages. Les personnages du roman mauricien tentent de conquérir ainsi leur ville au rythme du temps et des saisons.

\section{L'ordre difficile de la rue}

Espace plus ou moins ordonné, tantôt modelé sur le passé, tantôt façonné sur l'ordre neutralisant de la perspective, la rue témoigne à la fois d'un vécu et d'un désir de renouveau. Le héros mauricien la voit facilement sous ces deux aspects: celui qu'il reconnaît avec plaisir ou avec nostalgie et celui qu'il découvre. Dans Le Poids du jour, Robert M. Garneau ne retrouve plus sa paroisse natale de Louiseville, tant elle s'est transformée pendant ses dix-huit ans d'absence. Son désir tant de fois caressé de reconquérir ce coin de province, témoin d'une enfance malheureuse, s'évanouit dès «les abords du village devenu ville»; à la recherche des indices familiers qui orienteraient sa démarche, l'industriel montréalais constate la disparition des images visuelles de son enfance ${ }^{32}$. Quant à la «rue Principale d'autrefois, qui incarne peut-être à ses yeux le joug d'une tradition morale écrasante, Robert Garneau la voit toute modifiée: «c'est celle d'une ville et d'un chef-lieu. Toute droite et large entre les grands arbres dont on a abattu les pans pour dégager les affiches commerciales. Et une cohue d'autos de toutes couleurs, aux plaques de toutes les provinces; jusqu'à des Américains, cigare au bec dans l'encombrement des valises ${ }^{33}$. Impatient de prendre sa revanche sur cette ville qui lui rappelle sa condition d'enfant illégitime, Robert M. Garneau n'y retrouve plus l'ombre des fantômes qui peuplent encore son esprit : «ll avait même rêvé», nous dit le romancier, "qu'il passait seul, à pied, au milieu de la rue Principale tandis que, de chaque côté, immobiles et muets comme pour la Fête-Dieu, la foule des spectres sans visage formaient une double haie ${ }^{34}$."

Il en est de même pour Trois-Rivières dans Trente arpents. Dès les abords de la ville, Euchariste Moisan se sent «perdu». Vingt ans plus tôt, il était parvenu 
sans trop de difficultés à reconduire son fils aîné Oguinase au Séminaire. Mais en ce début de printemps 1920 , le paysan n'arrive pas à identifier la rue qui doit le mener chez son cousin Édouard: «... il se fût cru dans une capitale, à Québec ou même à Montréal, tant la ville s'était transformée. Il lui revint que, une douzaine d'années auparavant, une conflagration ${ }^{35}$ l'avait ravagée. Tout le cœur était maintenant un quartier aux voies larges, si larges qu'elles en paraissaient vides entre les prétentions parallèles des boutiques que surmontaient deux étages de briques multicolores ${ }^{36}$ ". Ainsi perdu au milieu de la cohue, isolé au sein d'une ville dont les signes les plus apparents ne lui disent plus rien, Euchariste Moisan fait quelque peu figure de déraciné, victime du progrès économique d'une ville en pleine évolution: au labyrinthe des ruelles d'autrefois, dont le tracé reste gravé dans sa mémoire, a succédé un aménagement urbain qui le déroute complètement.

L'isolement au sein de la ville peut aussi s'expliquer par une certaine hiérarchisation de la société. Ne gravit pas qui veut, en effet, les échelons conduisant au faîte de la pyramide sociale. La moindre faiblesse peut priver l'individu de tout avancement: «Dans les petites villes, notre vie ne nous appartient pas..." s'écrie I'héroïne de Mademoiselle Sérénité, "c'est la chose publique et elle reste soumise aux jugements des autres ${ }^{37}$ ". Plus encore, le sinueux parcours qui permet d'accéder au noyau privilégié d'une classe supérieure est si plein d'embûches que rares sont les personnages qui réussissent à vaincre les impitoyables règles du jeu et à passer d'un espace social à un autre. La ville que l'on pourrait croire accessible à tous, pratique un cloisonnement des espaces dont l'étanchéité empêche toute acclimatation normale: "L'atmosphère de la serre est fatale au lis. II y perd toujours son parfum et sa blancheur ", dira l'avocat Paul Des Marchets à I'héroïne du roman Madame Després, qui a «... gravi dans les larmes la pente abrupte d'un véritable calvaire ${ }^{38}$ " en voulant pénétrer dans l'espace bourgeois trifluvien; ses quinze ans de mariage avec le notaire Jean Després n'ont aucunement effacé aux yeux des dames de la caste son ascendance campagnarde. Enfin, nous assistons fréquemment au drame de personnages qui, après avoir tenté de résoudre maintes difficultés, prennent un récul provisoire face à leur ville pour la mieux maîtriser ensuite. Ils se réfugient temporairement dans des espaces plus vastes, soit à la campagne ou dans la forêt ${ }^{39}$, soit encore dans une métropole comme Montréal ${ }^{40}$ ou New York ${ }^{41}$ dans l'espoir de reconquérir leur petite ville dès leur retour.

\section{Le langage des affaires}

Malgré la verticalité de la structure sociale ${ }^{42}$, le héros mauricien ne renonce pas aisément à la quête de sa ville. L'appropriation de l'espace urbain demeure toujours à ses yeux un défi à relever, un espoir, une ambition à réaliser. Il est cependant curieux de constater que le langage économique de la ville lui parle peu. A l'exception de la proximité des usines de pâtes et papiers dont le message le touche de façon très affective, les activités commerciales et économiques le laissent presque indifférent. Ả son avis, le citadin qui s'enferme 
dans un bureau par une belle journée d'été se conduit comme un hurluberlu non initié à l'art de vivre : «Juillet laisse retomber sur la ville ses gerbes molles... Soudaine note fausse de l'homme qui descend de voiture, une serviette bourrée sous les bras, pour entrer dans un édifice où se font les affaires sans avoir seulement respiré l'été moins éphémère que lui ${ }^{43}$ ". Sans doute, les romanciers sont-ils les premiers responsables de cette quasi absence du monde de l'industrie et du commerce dans leur récit. Issus pour la plupart des professions libérales, ils connaissent assez mal le milieu des entreprises; peu intéressés à pénétrer dans les bureaux ou dans les usines, ils se contentent de faire le guet ${ }^{44}$ au coin de la rue, pour croquer sur le vif l'ouvrier ou l'employé à la sortie de son travail 45 .

En définitive, seule la banque jouit d'un certain prestige : occuper le poste de gérant de banque ou de comptable en chef est un signe de promotion sociale ${ }^{46}$. Mais une telle ambition révèle le désir d'accéder à un statut social supérieur plutôt que de manifester une réelle volonté de se rendre maître de l'espace économique de la ville. Derrière cet appétit d'avancement, on aspire à profiter de toutes les médiations culturelles que permet précisément tout statut social élevé: maison de campagne, voyage à l'étranger, résidence luxueuse, chalet en montagne...

\section{La fonction ludique de la rue}

La rue, espace ludique? Voilà peut-être le plus réel des spectacles auquel s'arrête le regard des romanciers. Riche de signes et de significations, la rue favorise un discours sans cesse orienté vers la communication, l'échange et l'appropriation de l'espace. Dans les quartiers populaires notamment, la rue devient le terrain de jeux tout indiqué pour les jeunes, à cette époque où l'organisation des loisirs et des sports est laissée à l'initiative de chacun: jeux de balle, de couteaux, de billes, jeux d'équipes, courses, sans parler des fugues et des mauvais tours. Aussi dans l'imaginaire du jeune citadin mauricien, habiter la même rue, voire le même quartier, revêt-il une grande importance: "j'étais son ami parce que j'avais son âge et que je demeurais sur sa rue ${ }^{47}$, confie le jeune narrateur de Pieds nus dans l'aube, à propos de son copain Fidor. Habiter la même rue, c'est en effet vivre ensemble, au jour le jour, des événements de toute nature qui laissent dans la mémoire des souvenirs impérissables.

Pour les adolescents mauriciens surtout, la rue devient ce «lieu de passage " qui mène à la conquête de la vie adulte; elle est à la fois tentations et séductions, rêves et appels, divertissements prohibés, rencontres nocturnes ou aventures passagères. Ainsi, les clubs, les bars, les restaurants et les gares jouent-ils un rôle de premier plan dans le roman mauricien. Bruyants et animés, ils sont une nécessité vitale pour une jeunesse qui cherche les moyens de liquider une fois pour toutes son adolescence pour accéder à l'aisance et à l'assurance des gens arrivés; l'anxiété et le désarroi, les émotions et les sentiments troubles s'y donnent régulièrement rendez-vous. Dans La Coupe 
vide, Adrienne Choquette décrit ainsi l'importance du restaurant «Handy Sweet » dans les allées et venues de quatre collégiens de province obsédés par le problème de la chair:

Assurément les quatre garçons, mains aux poches, pieds flâneurs, à la fois puérils et graves, naïs et avertis, qui parlaient sport et philosophie comme si le problème de la chair pour eux fût inexistant, assurément aucun des quatre n'avait clairement conscience du jeu redoutable commencé dans leur sang. En tout cas, pour rien au monde, Olivier Roch n'eût avoué certains réveils honteux après des rêves épuisants; ni François Rollin admis le trouble de sa chair devant la porte fermée de ses sœurs en arrière de laquelle à l'heure du coucher, se dérobait le mystère féminin. En ce moment d'ailleurs où certainement chacun rôdait encore en pensée aux alentours de Handy Sweet, qui eût pu déceler la vérité sous des réflexions hautement supérieures entrecoupées de baillements et de singeries déconcertantes... ${ }^{48}$.

Les lieux où se déroulent les activités ludiques des adultes présentent à peu près les mêmes caractéristiques. Ils renvoient presque toujours à un besoin de mobilité, de fuite ou d'entrain communicatif hors des espaces plus familiers. Qu'il s'agisse en effet du manège militaire à Trois-Rivières, du "Sandy Beach Inn » à la Pointe-du-Lac ou encore du Parc des Anglais à La Tuque, de tels lieux sont générateurs d'explorations, de promenades, de déplacements dans la ville ou hors de la ville; ils invitent le citadin agité, en mal de paix intérieure, à chercher dans la nuit urbaine les lieux de passage qui le mèneront au centre de lui-même. Aussi n'est-il pas surprenant de voir dans la rue le lieu de refuge par excellence contre l'autorité parentale, l'espace le plus propice pour échapper momentanément à la monotonie, à l'univers toujours clos du foyer ${ }^{49}$. En tout temps la rue est là, tout près, offrant gratuitement son espace à tous ceux qui refusent de se plier aux décrets de la tradition; certains personnages du roman mauricien ne l'oublient pas, qui demandent à la rue de les réconforter dans leur nuit ${ }^{50}$.

\section{Le plaisir de la rue}

Mais la rue est aussi une demeure, une sorte d'espace familial. On habite une rue, un peu comme on habite une maison. Aimer une ville, c'est d'abord aimer ses rues, ses avenues, ses boulevards, et même ses ruelles. Les personnages se sentent-ils à l'aise dans les rues de leurs villes?

La rue idéale, celle qui rallie davantage les goûts, les aspirations profondes des personnages, celle qui ravive leur désir d'y accéder, c'est la rue "plaisante», telle que décrite par Euchariste Moisan dans Trente arpents:

Les cousins habitent une rue calme, la rue Plaisante au nom joli dont la dut baptiser quelque premier propriétaire à l'âme poétique. L'œil ne voit, au départ, qu'un long jardin particulier clos d'une palissade que couronne en gerbe la frondaison des arbres; et les basses branches débordant la clôture inclinent librement vers le trottoir de brique rouge 
l'arceau de leurs feuilles, faisant une espèce de couloir ombreux pareil à un cloître... 51 .

Ainsi, la rue privilégiée évoque la végétation, la fraîcheur et la sérénité du jardin; tout enveloppée d'éléments naturels, elle favorise la libération des contraintes de la ville trépidante et fiévreuse. Voilà pourquoi certains personnages rêvent d'habiter une rue qui n'est pas reliée au centre démoniaque de la ville. Quelques-uns ont même le bonheur d'y passer leur enfance, comme le jeune Félix de Pieds nus dans l'aube:

Notre rue commençait dans les brouillards de la chute sauvage, sortait en grimpant d'une dense forêt, longeait la Vallée et s'en allait finir là-bas dans une coulée dangereuse, pays des souches et des lièvres.

Orgueilleuse avec ses trottoirs de bois, son chemin de sable, sa lumière coiffée d'un chapeau plat à tous les poteaux (qu'un homme à bicyclette, armé d'une perche venait allumer à chaque crécuspule), notre rue ne rentrait pas dans la ville. C'est pourquoi de nombreuses ruelles en goudron, étroites et bossues, affamées de poésie, venaient s'accrocher à elle comme des mendiantes 52 .

Dans les villes mauriciennes en plein essor, le citadin désire que sa rue reste un lieu où il fait bon vivre. Il ne souhaite nullement qu'elle vienne en tension avec la ville commerciale et industrielle. Qu'elle demeure plutôt un espace humain aussi authentique que fondamental, conservant la diversité de ses couleurs, de ses odeurs et de ses bruits, assumant à merveille son rôle de foyer d'adoption, réconciliant la parole du citoyen et le langage de la.ville...

\section{L'image de la ville désirée}

C'est encore dans la rue que le héros mauricien imagine la ville de ses rêves. En déambulant au gré de sa fantaisie dans les rues de sa petite ville de province surgit en lui l'image d'un habitat urbain qui contrarie l'usage de la ville. Requérant la ville comme un domaine collectif, il entend y vivre suivant les limites de son imagination créatrice. II rêve d'un espace urbain renouant ses rapports avec la nature, lui offrant les exubérances de la végétation et unissant le présent aux âges passés dans l'ultime besoin de s'identifier à son milieu natal.

\section{L'arrivée du printemps}

Les personnages du roman mauricien attendent toujours avec impatience les indices du réveil de la nature après un long hiver. La température devient plus clémente et le citadin de Trois-Rivières, de Shawinigan ou de Louiseville, éprouve une sensation de bien-être ou même d'euphorie contagieuse en savourant la douceur des premiers beaux jours de mai. II revoit enfin la terre, couverte de neige ${ }^{53}$ depuis six mois, il peut la toucher, en évaluer la densité et la fertilité. En déambulant au gré de ses fantaisies dans les rues de sa petite ville de province, il a le sentiment de renaître à la vie: "Et ce fut le printemps 
dans toute sa richesse de lumière caressante et d'odorante frondaison ${ }^{54}$, qui surprend l'héroïne de Jean Véron; un ravissement plus partagé dans La Coupe vide, où la redécouverte du printemps ravit une jeunesse en mal de liberté: «La fête est sur la ville, rayonne en vert. Ce n'est pas la lourde extase de juillet, mais une ivresse légère, à goût informulé d'aventure ${ }^{55}$ \%. Exaltation sensuelle d'une saison qui éblouit tous les regards, enchante l'odorat et permet au pied de fouler tout a son aise les trottoirs de bois!

En imposant son cycle d'éternel recommencement, le printemps triomphe partout. II ouvre les portes et les fenêtres pour rafraîchir et égayer l'intérieur des maisons. II favorise surtout, pour quelques mois, l'appropriation du paysage urbain; le citadin, alangui par le froid et la neige, trouve le temps de se déplacer. Il ambitionne de redécouvrir les lieux pittoresques les moins connus de sa petite ville. Parfois, c'est le goût de l'aventure qui le tenaille. qui le porte à observer, dans la campagne environnante, les prémices de la nature ${ }^{56}$, ou à satisfaire pleinement l'ivresse de ses sens en répondant à l'appel de la forêt sauvage ${ }^{57}$. Goûté comme un «vert d'espérance»58, le printemps ranime tous les espoirs; avec sa venue, l'espace urbain se déploie et gagne une dimension cosmique: il réintègre momentanément l'ordre du sacré.

\section{Les ilots de ruralité}

Certains lieux fixent encore le goût de la terre ancré dans le personnage mauricien. Ainsi le temps qu'il passe à se promener dans le parc public de sa petite ville lui apparaît toujours trop limité. II voudrait afficher son droit à la flânerie, y demeurer plus longtemps, même si la rue le rappelle à son travail quotidien ${ }^{59}$. Aussi, est-ce toujours avec une certaine gravité dans les gestes qu'il traîne ses pas sur le fin gravier des allées, s'arrête devant le spectacle d'un bassin d'eau, hume la fraîcheur des arbres, ou s'asseoit tout bonnement sur un banc pour mieux renouer contact avec la nature tout entière ${ }^{60}$. II en est de même lorsqu'il se rend au marché public de sa ville; là plus qu'ailleurs, il peut vérifier son appartenance vitale à la terre. La bousculade des gens venus faire provision de nourriture devient fraternelle; à l'espace agressif et anonyme de la rue se substitue un véritable centre horticole grâce auquel se rétablit la complémentarité de la ville et de la campagne jadis désertée ${ }^{61}$. Mais cette alliance est aussi fragile qu'éphémère: sa courte durée renforce le sentiment que la vie s'est réfugiée du côté de la "ruralité». Aller au marché, comme le fait François Barré dans La Campagne canadienne, c'est vouloir revenir à la ville comme on revient à son sol natal, en oubliant la domination subtile de la campagne environnante par la ville ${ }^{62}$.

Ce besoin instinctif de se référer à la nature met en évidence la prédilection du personnage mauricien pour un lieu de résidence plus rustique qu'urbain. C'est en effet à la périphérie du centre urbain que l'espace lui paraît le plus familier et le plus original. Le jeune héros de Pieds nus dans l'aube avoue par exemple sa fierté de voir la maison tourner «carrément le dos à la 
population et à la ville..."; "face à la Vallée», ajoute-t-il, elle "fixait comme en extase la lointaine caravane des monts bleus... ${ }^{63}$. L'héroïne de Mademoiselle Sérénité éprouve un sentiment similaire à la vue du long boulevard longeant le Saint-Maurice à Shawinigan; non seulement "cette élégante allée de béton" lui apparaît-elle comme un lieu de rêve pour y élire domicile, mais elle semble tout particulièrement l'inviter à un "voyage interminable» vers la grande nature mauricienne ${ }^{64}$. Plus encore, la ville rêvée, l'anti-ville, serait celle où domineraient les richesses d'une nature protégée, une ville sensible aux attentes des citoyens, en harmonie avec son environnement et, par-dessus tout, capable d'organiser elle-même des espaces respectant les nuances de la nature ${ }^{65}$ : «... une ville en pleines montagnes»66, voilà l'aménagement urbain dont rêve plus d'un personnage mauricien.

\section{La nostalgie du passé}

A l'aspiration de vivre en harmonie avec la nature s'ajoute enfin la volonté de conserver intact le visage historique de la ville. Le modernisme ne suscite guère d'enthousiasme chez la plupart des personnages. La circulation rapide et intense des véhicules irrite leur sensibilité et leur fait regretter le temps où le rythme de la vie était plus lent, plus pastoral: nostalgie d'une époque où le citadin avait le loisir d'arrêter sa voiture au beau milieu de la chaussée pour faire un brin de causette avec un ami, sans risquer d'être sèchement interpellé par un agent de police ${ }^{67}$. Plus encore, la nécessité de démolir de vieilles maisons pour construire à neuf provoque une réaction défavorable contre une urbanisation déchaînée, envahissante et irrespectueuse de la tradition. Abattre des pans d'arbres «pour dégager les affiches commerciales" 68 , c'est détruire la vie et l'œuvre des aînés; démolir un vieux et sympathique trottoir de bois afin de le remplacer par un autre en ciment, c'est effacer les traces creusées jadis par les premiers bâtisseurs ${ }^{69}$; transformer une ancienne rue sinueuse et attrayante pour les piétons en une voie droite et impersonnelle, c'est réduire l'usage collectif de la ville ${ }^{70}$. En somme, rompre avec un passé heureux, c'est désavouer une époque que pourrait dénoncer ou du moins ralentir la mise en place d'un ordre urbain différent. La «politique du progrès» réjouit par ailleurs toute une jeunesse qui voit l'avenir de la ville lui appartenir: «l'invention, l'avancement, l'ordre, voilà ce qu'il nous faut...! Dehors les vieilleries " "lance un jeune de La Tuque enthousiasmé par le "vent de prospérité et d'orgueil» qui souffle sur sa petite rue. Pour l'ensemble des personnages, le phénomène de l'urbanisation signifie cependant la perte d'un espace investi grâce auquel la ville manifestait encore sa permanence; leur attachement à leur ville renvoie instinctivement à une image qui s'estompe, arrêtée dans le temps, ou encore réduite aux apparences extérieures.

\section{Conclusion}

Au terme de cette étude sur le discours romanesque et le discours urbain, certaines questions plus générales se posent. Les romanciers retenus aux 
fins de cette analyse nous donnent-ils une juste perception du paysage urbain de la Mauricie? A quels ressorts du langage romanesque font-ils principalement appel dans leur représentation du discours sur la ville? Quelle logique de l'imaginaire cherchent-ils, intentionnellement ou invölontairement, à établir, à «spatialiser», comme si elle était le recours définitif contre la ville elle-même? Autant d'interrogations qui nous paraissent d'abord liées à l'espace du roman, lui-même imprégné de subjectivité, limité par le «point de vue» du narrateur, chargé d'états $d^{\prime}$ âme tout aussi fictifs que les personnages qui les ressentent.

Au premier regard, cette douzaine de romans, parus entre 1925 et 1950. ne semble aucunement représentative d'un discours possible sur la ville. Peut-on en effet faire temporairement abstraction des circonstances particulières entourant leur rédaction, renoncer à l'étude de leurs sources et éviter le fait que tous n'ont ni la même structure spatiale, ni la même valeur esthétique. Des treize romans publiés au cours de ce quart de siècle, six seulement ont pour cadre romanesque une des villes importantes de la Mauricie ${ }^{72}$. Quant aux sept autres, ils ont comme espace principal soit la campagne $^{73}$ soit la forêt ${ }^{74}$, et décrivent la ville mauricienne en mettant particulièrement l'accent sur ses lieux les plus symboliques. En d'autres termes, est-on en droit de considérer ces romans comme un texte unique renvoyant à un même espace géographique?

Un fait est d'abord remarquable: tous puisent abondamment leur inspiration dans le paysage physique et culturel de la Mauricie ${ }^{75}$. En ce sens, ils peuvent être qualifiés d'œuvres régionales; ils le sont d'autant plus que la majorité d'entre eux se concentrent autour de certains espaces particuliers, s'attachent à les mettre en opposition les uns par rapport aux autres, quadrillant ainsi littéralement le territoire sans toutefois s'appliquer à le décrire dans sa totalité. Autre élément particulier de ce corpus de romans: il est antérieur aux années 1950, époque au cours de laquelle les principales villes de la Mauricie ont subi une mutation qui caractérise leur espace urbain d'aujourd'hui. Plus encore, la chronologie interne de chacun de ces romans nous apprend que huit d'entre eux décrivent l'espace mauricien des années 1900 à 1925 alors que les cinq autres situent cet espace entre les années 1925 et 1935. C'est donc dire l'importance accordée à un temps marqué par le passé, par la réminiscence d'images qui fixent l'espace urbain dans une sorte de temps continu. Une telle contemplation du passé urbain n'est cependant pas dénuée d'enseignement; elle met en évidence le désir des romanciers d'actualiser davantage les changements que subissent leurs petites villes de province au moment où elles leur servent de lieux d'inspiration. Plus que du temps révolu, c'est de celui de leur époque que la plupart d'entre eux veulent rendre compte: un temps marqué par l'asservissement de l'espace urbain au pouvoir économique.

Au discours de la ville longtemps façonné par le sacré s'est donc peu à peu substitué un langage morcelé de signes industriels; à l'église, temple de pierres, s'est timidement ajoutée l'usine de béton; devenue peu à peu 
une sorte de "cathédrale laïque " 76 , elle a fini par remplacer celle-là, amenant avec elle la multiplication des codes urbains. Autrement dit, les romanciers mauriciens, du moins ceux étudiés ici, ne font que reproduire un langage urbain déjà institué, une rhétorique qui a perdu sa vertu imageante parce que déjà codifiée dans le vécu de la ville. Hormis Adrienne Choquette qui sort des sentiers battus, rares sont en effet les auteurs qui transgressent les propriétés du symbolisme urbain qui s'imposent à eux; de La Campagne canadienne (1925) d'Adélard Dugré au Poids du jour (1949) de Ringuet, c'est invariablement les mêmes polarités spatiales qui nous sont présentées, les mêmes récurrences des lignes verticales et horizontales qui déterminent la démarche des personnages: à la cheminée de l'usine s'oppose le clocher de l'église paroissiale; à la turbulence du centre urbain, la douceur du foyer familial; à l'ordre de la rue, la route menant à la campagne; à la ville usinière, la banlieue printanière.

Un tel langage est davantage vécu qu'inventé de toutes pièces. II est d'abord issu du désir et de l'inaccomplissement. Sans doute - et nous l'avons souligné - demeure-t-il au premier chef un discours verbal, déterminé par les mots du récit, allant jusqu'à se soumettre aux lois du genre. Mais s'il naît d'une mosaïque de mots imaginée par l'écrivain, il n'en demeure pas moins qu'il affirme la présence du monde ${ }^{77}$. Disons-le autrement. Si, par son récit, l'écrivain exprime un possible du réel, c'est-à-dire un discours capable d'avènement et donc porteur d'un sens, le réel qui émane de l'œuvre est tout entier ouvert aux dimensions du monde: la forme représentante du réel est ancrée dans l'imaginaire du roman, comme l'est l'imagination créatrice. $\mathrm{Vu}$ ainsi, le discours que tiennent les romanciers mauriciens sur leur ville

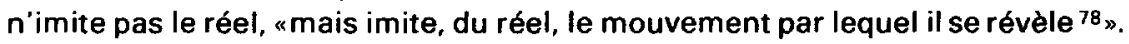
Or, ce mouvement est bien celui de la production et de l'exploitation industrielles; tel un scénario symbolique, il envahit la ville, lui impose son ordre de grandeur, et force plus d'un romancier à décrire ou à contester son irruption dans les moindres lieux de la ville. Ainsi s'éclaire la signification des étapes que nous avons suivies: elles se sont fixées à même cet échange obligé entre deux discours, l'un et l'autre se désignant sans jamais se confondre: ascendance symbolique des lieux de prière et des lieux industriels dévoilant devant les yeux des romanciers l'environnement total de la ville ${ }^{79}$ : culture, travail, habitat...; communicabilité des signes urbains, de la rue surtout qui ne cesse d'être décrite, comme si elle offrait à l'écrivain et à ses personnages l'unique possibilité d'échanges et de rencontres, dans leur déplacement à travers leur petite ville de province; enfin, image rêvée de la ville, qui surgit comme le symbolisme religieux ou industriel du passé urbain, mais pour figurer à l'inverse des lieux symboliques, l'avenir de la ville.

Que l'image la plus archaïque soit à la source d'une vision rêvée de la ville, voilà qui n'a rien d'extraordinaire! Elle s'impose comme un absolu avec lequel tout commence ou recommence: le printemps, le jardin, le site historique, trois origines mythiques qui marquent le temps et l'espace. Contre la ville elle-même donc, contre son fonctionnalisme, les romanciers opposent 
le geste antique d'appropriation de l'espace urbain. Sans doute y a-t-il dans une telle actualisation du passé plus d'imaginaire que d'imaginatif, plus d'enracinement dans l'inconscient collectif que de visée personnelle vers l'avenir, plus de refus que d'exigence. N'empêche que les signes de la nature sont nombreux, plus nombreux que ceux de l'histoire; les uns et les autres apparaissent intimement liés, décrits dans leur dernier état spatial, consciemment actualisés, sinon par les romanciers qui en tracent les contours, du moins par les personnages qui se plaisent à les détecter et à les revoir. Mais la multiplication de ces signes pourrait tout aussi bien signifier qu'ils se font de plus en plus rares dans le paysage urbain, d'autant plus rares même que le droit d'en jouir librement se retrouve du côté des groupes dominants. Dualité de l'imaginaire? Tantôt retouché, épuré, altéré par l'idéologie dominante, tantôt transgressé par l'écrivain lui-même lorsque, acceptant à la limite de le traduire, il se trouve à le dénoncer par la praxis même d'un art qui lui échappe. Ainsi, cette fuite vers la nature et vers l'histoire témoigne d'une incapacité de s'approprier l'espace urbain. Dans aucun des romans étudiés, les personnages ne nous paraissent pleinement satisfaits de la qualité de leur ville de province; ils ont le sentiment qu'elle ne leur renvoie plus qu'un seul message: celui du fonctionnalisme urbain. Qu'ils aient alors envie de lui tourner parfois le dos, de remonter le cours du temps pour retrouver son lieu originaire, voilà bien une preuve que tout discours - romanesque ou urbain - est d'abord possession, pour l'un possession de la terre, et pour l'autre possession de notre être.

1. Cet article est issu d'un ensemble d'analyses commandées par un projet de recherche actuellement en cours sur \& la Mauricie et ses romanciers». Bénéficiant de subventions provenant du ministère de l'Éducation (programme de Formation des chercheurs et d'action concertée), du Conseil de recherche en sciences humaines du Canada et du Fonds institutionnel de recherche de I'Université du Québec à Trois-Rivières, ce projet vise à dégager, à décrire et à analyser les composantes dynamiques de l'espace littéraire mauricien à travers un corpus de soixante-cinq romans québécois parus entre 1850 et 1950 , et écrits, soit par des romanciers de la région elle-même, soit par des romanciers de l'extérieur, mais qui ont campé entièrement ou partiellement l'action de leur(s) récit(s) en Mauricie.

2. Les images de la ville, Paris, éditions Anthropos, 1973, pp. 18 et 42.

3. "L'histoire littéraire et la sociologie», conférence faite à l'École des Hautes Études sociales le 29 janvier 1904; reproduite dans Essais de méthode de critique et d'histoire littéraire, rassemblés et présentés par Henri Peyre, Paris, Librairie Hachette, 1965, pp. 61-80.

4. Les paysages urbains, Paris, Armand Colin, 1973, p. 8.

5. La majorité des auteurs de ces romans sont eux-mêmes natifs de la Mauricie: Hervé Biron, Nuages sur les brûlés, Montréal, éditions Fernand Pilon, 1947. 207 p.: Adrienne Choquette, La Coupe vide (1948), Notre-Dame des Laurentides, les Presses Laurentiennes, 3e édition, 1978, 222 p.; Un jeune de 73, (abbé Joseph Desaulniers), "Marie I'Espérance, scènes de la vie du Saint-Maurice", dans Le Bien public, du 11 février au 23 juin 1932; Adélard Dugré, s.j., La Campagne 
canadienne, Montréal, Imprimerie du Messager, 1925, 235 p.; Moïsette Olier (Corinne Garceau), L'Homme à la physionomie macabre, Montréal, éditions Edouard Garand, 1927, 145 p. et Mademoiselle Sérénité. Trois-Rivières, Le Nouvelliste, 1936, 212 p.; Jean Véron (abbé Eddie Hamelin), Madame Després, TroisRivières, éditions du Bien public, 1934, 190 p.; Félix Leclerc, Pieds nus dans l'aube (1946), Montréal, éditions Fides, «Bibliothèque canadienne-française", 1971, 215 p.; Ringuet (Philippe Panneton), Trente arpents (1938), Montréal, éditions Fides, Collection du Nénuphar, 1965, 306 p. et Le Poids du Jour. Montréal, éditions Variétés, 1949, 410 p.; Marcel Trudel, Vézine, Montréal, éditions Fides, 1946, $264 \mathrm{p}$. Nous avons également ajouté à cette liste deux autres romanciers qui ont adopté la Mauricie comme source d'inspiration de leur œuvre: Marie Lefranc, La Randonnée passionnée (1936). Montréal, éditions Fides, Collection du Nénuphar, 1962, 159 p.: Adolphe Nantel, A la hache, Montréal, éditions Albert Lévesque, 1932, $234 \mathrm{p}$.

6. Trente arpents, p. 97.

7. La Campagne canadienne, p. 128.

8. Madame Després, p. 128.

9. C'est ce réseau symbolique que voit François Barré dans La Campagne canadienne, au moment où il contemple Trois-Rivières du haut de ses côteaux: «En bas, le Saint-Maurice, sombre et tumultueux, se précipitait sous les grands ponts pour mêler ses eaux noires aux eaux verdâtres du grand fleuve. Au-delà des eaux, c'était la côte sud et la lignée des blanches églises qui se mirent au rivage. Très loin, à droite, par-delà les miroitements du lac Saint-Pierre, c'était l'église de la Baie-du-Febvre, dont il reconnaissait bien la toiture étincelante, si facilement visible de la Pointe-du-Lac. Puis venait Nicolet, dressant les fiers clochers de sa cathédrale, puis Sainte-Angèle, en face, puis Bécancour, Gentilly, et même les Becquets, là-bas, au fond de la grande baie. Sur la côte nord, Batiscan. Champlain, le Cap, autrefois simple amoncellement de pauvres habitations ouvrières, aujourd'hui petite cité industrielle, pleine d'activités, tout empanachée de la fumée des usines qui bordent l'embouchure du Saint-Maurice, mais pieuse quand même et recueillie, étalant avec amour la simplicité de son vieux sanctuaire* (pp. 208-209). Joseph Desaulniers reprend pratiquement la même vision dans Marie L'Espérance, scènes de la vie du Saint-Maurice: I"héroïne de son roman, sur le talus du «Platon», admire les clochers des églises qui dominent de leurs flèches élancées le paysage environnant. (Le Bien public, 26 mai 1932, p. 5).

10. Voir tout particulièrement la description de l'ancienne église de l'ImmaculéeConception de Trois-Rivières dans Marie L'Espérance, scènes de la vie du SaintMaurice, de Joseph Desaulniers. Le romancier décrit ainsi la voûte de l'église: "La forme de la voûte, qui s'adaptait admirablement aux exigences de notre climat, datait de loin. Elle avait été emportée de France, dès les premiers temps de la colonie. Cette voûte n'était pas seulement une forme, elle était aussi un symbole. Elle s'élevait vers le Ciel, d'abord rapidement pour représenter l'élan du coeur et l'ardeur de la prière. Puis, elle ralentissait sa course, montait encore, mais sans se hâter, et finissait par s'immobiliser au centre. La prière s'était levée vers le Ciel et le Ciel semblait s'être rapproché pour la recueillir plus vite et intimer qu'elle avait été entendue. (Le Bien public, 31 mai 1932, p. 5).

11. Madame Després, p. 89.

12. Ibid., p. 219. Voir aussi La Campagne canadienne, p. 209.

13. Madame Després, p. 129.

14. Madame Després, pp. 131-132. Voir aussi, Pieds nus dans laube, pp. 169-172; Trente arpents, p. 196: Marie L'Espérance.... op. cit., 12 mars 1932, p. 5.

15. La Randonnée passionnée, p. 113.

16. Madame Després, pp. 131-132.

17. Trente arpents, pp. 104-105. A un degré moindre, le petit collège de La Tuque présente les mêmes caractéristiques. École d'éducation supérieure, c'est aussi un lieu où l'autorité et la discipline sont des valeurs premières. Pieds nus dans laube de Félix Leclerc a bien rendu, en quelques traits, l'image que le collège 
projette dans la mémoire des jeunes de l'endroit: «... petit collège de briques où sont les pupitres vernis, le tableau noir, le maître et les cahiers* (p. 150).

18. Pieds nus dans l'aube, pp. 150 et 169.

19. La Coupe vide, p. 43.

20. Voir à ce propos les très justes notations de Marcel Trudel dans Vézine. En quelques pages, le romancier décrit la vie des collèges, à Trois-Rivières, au temps où les étudiants portaient l'uniforme, "cașquette militaire sur la tête», vêtus "d'un surtout noir» et «la taille serrée d'un ceinturon vert» (pp. 220-234).

21. Les hôpitaux pourraient encore s'ajouter à cette liste de monuments symboliques. Leur architecture extérieure, un peu comme celle des églises, est imposante et incarne la tradition chrétienne: I'hôpital Saint-Joseph de Trois-Rivières dresse fièrement «ses multiples tourelles et son mince clocher ( La Campagne canadienne, p. 213); celui de La Tuque «...bien assis sur des roches, surplombe la vallée» mauricienne (Pieds nus dans l'aube, p. 21). La présence à l'intérieur de l'hôpital de religieuses, d'infirmières et de médecins, confère à cet espace un caractère androgyne. Mais - et c'est là l'essentiel - l'horreur instinctive qu'inspire la maladie brouille les images et les sensations. La propreté aseptique devient froideur, l'organisation et l'efficacité sont perçues comme des signes d'indifférence ou d'insensibilité. L'hôpital exprime à un très haut degré le drame de la solitude face à la souffrance et à la mort (Trente arpents, pp. 194-195).

22. La Campagne canadienne, p. 65; voir aussi Madame Després, p. 218.

23. Pieds nus dans l'aube, p. 214.

24. Lbid., p. 193. Leclerc écrit encore: «La ville, c'est l'attente, la cloche, la sonnerie, le sifflet qui te dit: «Lève-toi, viens-là, puis fais ceci, va dîner; c'est tout, bonsoir”. Et ça recommence interminablement. La ville, c'est un immense cri que personne n'entend; c'est un lourd silence roulant des bruits insupportables... La ville, c'est... des milliers de mains tendues par en haut qui prient. Des milliers de muscles qui travaillent. Des bribes d'angélus perdues dans le rire des cabarets. Des millions de mâchoires fermées qui souffrent. C'est un bruit de ferraille, la vapeur pourrie qui sort des caves et sent mauvais... C'est la vallée de larmes i».

25. Ibid., p. 20.

26. La Randonnée passionnée. 1961, p. 112; voir aussi Hervé Biron, Nuages sur les brülés, 1947, p. 65.

27. A la hache, pp. 135 et 163.

28. La Randonnée passionnée, p. 112. Voir aussi pp. 135 et 145.

29. Trente arpents, p. 192.

30. Ibid.

31. Pieds nus dans l'aube. pp. 20, 46, 193 et 194.

32. Ringuet décrit ainsi l'arrivée de son héros dans sa ville natale: «... il ne pouvait imaginer les rues autrement que telles il les avait jadis connues. Pour lui, elles étaient fixées dans le temps et dans une forme éternelle, semblables à ces photos passées où hommes et choses ont été arrêtés, gelés instantanément par le public. Semblables, ces rues, à celles qui penchaient les gouttières basses de leurs toits garnis d'une barbe de glaçons sur un enfant passant qui s'appelait Michel». (Le Poids du jour, pp. 215-220).

33. Le Poids du jour, p. 220.

34. Ibid.

35. Le 22 juin 1908, un incendie détruisit le centre-ville de Trois-Rivières.

36. Trente arpents, p. 192. Voir aussi le roman de Marcel Trudel, Vézine, où le héros ne reconnaît plus Trois-Rivières, tant elle s'est agrandie depuis 1908: «... la ville s'était joliment développée : elle s'était allongée vers le nord et garnissait le premier coteau; de grosses usines couvraient d'immenses terrains; des églises avaient surgi; la cour du séminaire, qui autrefois donnait sur la campagne, se trouvait perdue au milieu de la ville» (p. 220).

37. Mademoiselle Sérénité, p. 20. 
38. Madame Després, pp. 66-75. Le point de vue de l'avocat Des Marchets, qui est celui du romancier, est révélateur à plus d'un titre; ses réflexions sur la solidité des barrières sociales ne font que mieux ressortir les réactions de rejet dont est victime l'héroïne du roman: «Je vois, madame, malgré vos belles qualités et votre ambiion, que vous n'avez pas réussi à vaincre les préjugés de la noblesse contre la bourgeoisie, des grands contre les petits, de la ville contre la campagne, comme vous voudrez.*

39. L'Homme à la physionomie macabre, p. 123; voir aussi Mademoiselle Sérénité, pp. 53-57.

40. Le Poids du jour, pp. 157-158.

41. Mademoiselle Sérénité, pp. 103-105.

42. Dans Le Poids du jour, Ringuet décrit ainsi les années d'enfance de Robert-Michel Garneau à Louiseville: «ll avait suivi le chemin banal, ne s'en écartant point, ramené d'ailleurs dans les ornières normales par des mains vigilantes chaque fois qu'il eût voulu tirer vers la libre campagne" (p. 34).

43. La Coupe vide, p. 43.

44. Ibid., p. 21.

45. A l'instar des édifices à bureaux et des usines, les petites boutiques sont asphyxiantes, privées de soleil et d'air pur. Qu'il s'agisse de la boutique du bimbelotier, du marchand de tabac, du sellier, elle est généralement basse, obscure et étouffante. Parfois, cependant, elle fait figure d'espace sympathique dans le décor urbain; avec elle, nous gagnons le terrain de la vie familière: c'est un lieu de rencontres, de contacts humains agréables et chaleureux, où l'intensité de la fraternité fait oublier la laideur des lieux. Plus que les édifices publics, la boutique retient le regard du romancier, qui s'attarde longuement à décrire le décor et les gestes du marchand.

46. Le Poids du jour, pp. 94-96; Madame Després, p. 158.

47. Pieds nus dans l'aube, p. 44.

48. La Coupe vide, p. 25; voir aussi pp. 17 et 24.

49. Il faut particulièrement noter l'importance des "dancings» comme lieux d'amusement les plus contestés de l'époque. Dans L'Homme à la physionomie macabre. Moïsette Olier met clairement en évidence les remous que suscite dans la population la dénonciation «par le clergé trifluvien» de l'auberge "Sandy Beach Inn * à la Pointe-du-Lac (pp. 50-52).

50. Mais le mouvement vers le cercle familial est aussi mis en évidence par les romanciers. Les expressions «gagner le foyer», a rentrer à la maison", «retourner chez soi», sont habituelles pour la plupart des personnages; riche ou paurre, chacun est heureux d'avoir un toit, de se retrouver parmi les siens. La chambre, surtout, assure un refuge contre la délation de la rue; elle est le lieu de prise en charge de soi, ou encore le "double de soi le plus souvent approbateur", comme I'indique Géraldine Rollin, dans La Coupe vide, (p. 62); voir aussi Mademoiselle Sérénité, pp. 21-23, Pieds nus dans l'aube, p. 77 et Madame Després, p. 62.

51. Trente arpents, p. 100.

52. Pieds nus dans l'aube, p. 19.

53. L'hiver n'est cependant pas percu par tous comme une saison déprimante et difficile à supporter. Ainsi, dans Nuages sur les brûlés d'Hervé Biron, l'enlèvement de la neige dans les rues de la ville représente pour une certaine catégorie de travailleurs I'unique possibilité de gagner quelques sous: «... Plante et Hamelin passaient leur hiver à Shawinigan, heureux de semaine en semaine si de bonnes bordées de neige venaient remplir les rues et leur donner l'occasion de gagner au pelletage de quoi nourrir leur famille. Mais que la terre restait nue jusqu'aux Rois, quelle détresse! Les enfants et les mères priaient l'Enfant-Jésus de faire neiger durant les Avents, afin d'avoir un peu d'argent pour préparer le réveillon de Noël et acheter quelques jouets nouveaux* (p. 50).

54. Madame Després, p. 152.

55. La Coupe vide, p. 21. 
56. Le Poids du jour, p. 85, voir aussi Madame Després, p. 153.

57. Pieds nus dans l'aube, pp. 157-158.

58. Madame Després, p. 152.

59. La Coupe vide, p. 21; voir aussi La Campagne canadienne, p. 193.

60. La Coupe vide, pp. 33-34; voir aussi Mademoiselle Serénité, p. 43.

61. La Campagne canadienne, pp. 183-184.

62. Ibid., p. 182.

63. Pieds nus dans laube, p. 15.

64. Mademoiselle Sérénité, p. 44.

65. L'Homme à la physionomie macabre, p. 149.

66. Ibid., p. 150.

67. Le Poids du jour, p. 219, voir aussi Trente arpents, pp. 193.

68. Le Poids du jour, p. 220.

69. Pieds nus dans l'aube, pp. 177-178.

70. Trente arpents, p. 192.

71. Pieds nus dans l'aube, pp. 177-179.

72. A ce titre, Trois-Rivières est la ville qui a inspiré le plus de romans : Madame Després de l'abbé Eddie Hamelin, Mademoiselle Sérénité de Moïsette Olier et La Coupe vide d'Adrienne Choquette. Puis viennent Shawinigan dans L'Homme à la physionomie macabre de Moísette Olier, La Tuque avec Pieds nus dans l'aube de Félix Leclerc et Louiseville dans Le Poids du jour de Ringuet.

73. Trois romanciers adoptent principalement le milieu paysan ou campagnard comme lieu d'action de leurs personnages: Adélard Dugré situe l'intrigue de La Campagne canadienne à la Pointe-du-Lac. Ringuet celle des Trente arpents aux environs de Maskinongé, et Marcel Trudel celle de Vézine à Saint-Narcisse, son village natal.

74. Quant à la forêt mauricienne, elle constitue l'espace principal des récits suivants : La Randonnée passionnée de Marie LeFranc, Marie L'Espérance... de l'abbé Joseph Desaulniers, A la hache d'Adolphe Nantel et Nuages sur les brülés d'Hervé Biron.

75. Ils ne sont pas d'ailleurs les seuls. Sur un total de trente romans, parus entre 1850 et 1950, dont l'action se passe entièrement en Mauricie, dix-neuf ont été écrits par des romanciers natifs de la région et onze proviennent d'écrivains québécois ou étrangers qui ont vécu en Mauricie ou ont adopté cette région québécoise comme cadre d'action de leurs ceuvres. A ce nombre, il faut encore ajouter un total de trente-trois romans québécois, parus au cours de la même période, qui évoquent partiellement un ou plusieurs espaces caractéristiques de la Mauricie.

76. G.C. Argan, cité par Gillo Dorfles, Mythes et rites d'aujourd'hui, Paris, Klincksieck, 1975 , p. 255, note 5 .

77. Voir à ce sujet Jean Weisgerber, L'espace romanesque, Lausanne, éditions L'Âge d'homme, 1978, 268 p. L'auteur définit ainsi l'espace du roman: aL'espace du récit englobera donc des représentations mentales, le milieu psychique - illimité en théorie - des songes, des passions et de la pensée, et d'autre part, des sensations et perceptions occasionnées par un monde physique dont la finitude est d'ordinaire aussi évidente que celle du sujet qui s'y meut."

78. Mikel Dufresne, Esthétique et Philosophie, Paris, éditions Klincksieck, tome 2. 1976, p. 62.

79. Pierre Sansot, Poétique de la ville, Paris, éditions Klincksieck, 1971, p. 23. Pour la symbolique des grands lieux urbains, voir aussi Henri Lefebvre, Critique de la vie quotidienne, Paris, éditions de L'Arche, 1961, tome 2, pp. 279-314 et Raymond Ledrut, op. cit., pp. 142-154. 